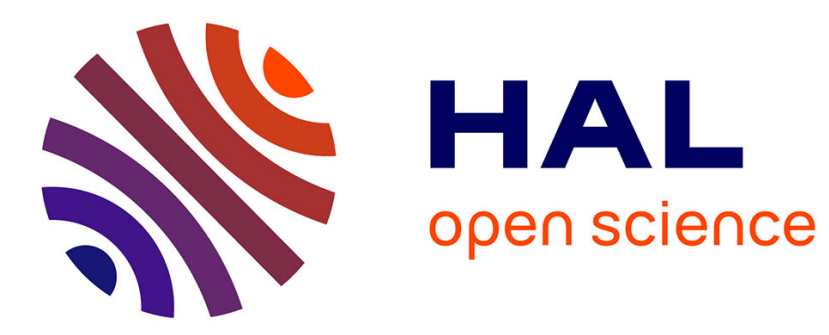

\title{
Mieux comprendre les difficultés d'une reprise externe grâce à l'approche des représentations sociales
}

\author{
Fabienne Bornard, Catherine Thevenard-Puthod
}

\section{To cite this version:}

Fabienne Bornard, Catherine Thevenard-Puthod. Mieux comprendre les difficultés d'une reprise externe grâce à l'approche des représentations sociales. Revue Internationale PME, 2009, 22 (3-4). halshs-01492656

\section{HAL Id: halshs-01492656 \\ https://shs.hal.science/halshs-01492656}

Submitted on 20 Mar 2017

HAL is a multi-disciplinary open access archive for the deposit and dissemination of scientific research documents, whether they are published or not. The documents may come from teaching and research institutions in France or abroad, or from public or private research centers.
L'archive ouverte pluridisciplinaire HAL, est destinée au dépôt et à la diffusion de documents scientifiques de niveau recherche, publiés ou non, émanant des établissements d'enseignement et de recherche français ou étrangers, des laboratoires publics ou privés. 


\title{
Mieux comprendre les difficultés d'une reprise externe grâce à l'approche des représentations sociales
}

\author{
Fabienne Bornard - ESC Chambéry - IREGE (Université de Savoie) \\ Catherine Thévenard-Puthod - IREGE (Université de Savoie)
}

\section{INTRODUCTION}

Le «repreneuriat» représente en France ${ }^{1}$ un enjeu de taille, puisque près de 900000 entreprises seront vraisemblablement à céder dans les dix ans à venir (30 à 40\% des dirigeants ayant plus de 50 ans en 2004, selon une enquête de la CRCI Rhône-Alpes, 2006). Bien que la sensibilisation des différents acteurs économiques à la nécessité de préserver ces structures existantes ait été amorcée depuis plusieurs années déjà, la situation demeure alarmante. Ainsi le nombre d'entreprises reprises est faible et en diminution constante (40 000 reprises par an au début des années 2000, contre 60000 en 1987, selon Oséo Bdpme, 2005). En outre, il apparaît que la signature d'un acte de vente entre un cédant et un repreneur ne soit pas un indicateur de réussite de l'opération, puisqu'une reprise sur cinq échoue avant six ans. Ce taux d'échec augmente si le repreneur est un tiers extérieur à l'entreprise acquise, ce qui est de plus en plus souvent le cas, car près d'une entreprise sur deux est cédée à un repreneur externe (Oséo Bdpme, 2005). Il apparait donc important d'analyser les raisons qui peuvent expliquer ces échecs, tant en amont (l'absence de transaction) qu'en aval du processus repreneurial (c'est-à-dire une fois que la transaction a été réalisée).

Les premiers travaux académiques sur le repreneuriat se sont attachés à relater les freins empêchant le bon déroulement de ces transactions : opacité du marché (Chaineau, 1989; Vatteville, 1994), fiscalité pénalisante (Baumert, 1992), insolvabilité des repreneurs (Lehman, 1993)... A la suite de ces réflexions, de nombreuses initiatives institutionnelles se sont développées pour palier ces problèmes : création de bases de données de cédants/repreneurs, aides financières de l'Etat et accompagnement d'OSEO Sofaris, etc. Cependant ces dispositifs, centrés sur les aspects «techniques » du repreneuriat, ne lèvent qu'une partie des obstacles. Ils laissent en effet de côté la dimension psychologique entourant l'opération, qui peut pourtant fortement perturber - voire empêcher- le bon déroulement d'une reprise. Ce n'est que récemment que la littérature en repreneuriat s'est intéressée à ces aspects

\footnotetext{
${ }^{1}$ Cet enjeu se mesure également à l'échelle européenne, puisqu'un tiers des entreprises de l'Europe Occidentale devrait changer de propriétaires dans les dix prochaines années (CRCI Rhône Alpes, 2006).
} 
psychologiques (Pailot, 1999 ; Meier, 2002 ; Bah, 2009²), en mobilisant notamment la théorie du deuil et en se centrant sur les problèmes en amont du processus, c'est-à-dire sur les difficultés du cédant à « lâcher-prise ».

Dans la lignée de ces derniers travaux, cet article propose d'expliquer une partie des échecs des reprises, en mettant en avant les facteurs subjectifs et intersubjectifs qui influencent le processus repreneurial. Dans une perspective sociocognitive des organisations, nous pensons que la compréhension du comportement des principaux protagonistes d'une reprise (cédant, repreneur, salariés...) doit passer par celle de leur modèle cognitif. Nous avons pour cela choisi d'utiliser le concept de représentation sociale qui, alors même que son intérêt a été souligné en entrepreneuriat (Verstraete, 2003 ; Cusin et Fayolle, 2005), n'a pas encore, à notre connaissance, été mobilisé par les chercheurs dans le domaine du repreneuriat. L'originalité du concept de représentation sociale est qu'il permet d'englober à la fois toutes les phases du processus (tant en amont qu'en aval) et l'ensemble des acteurs concernés : cédant, certes, mais aussi repreneur, salariés et parties prenantes externes à l'entreprise. Il permet en outre d'apporter un éclairage sur les conduites individuelles, tout en prenant en compte leur dimension collective (ce qui est intéressant dans le cas des salariés par exemple). Enfin, audelà de son attrait conceptuel, il permet de proposer aux professionnels des pistes d'accompagnement qui pourraient faciliter le déroulement d'une reprise externe par une meilleure prise en compte des difficultés cognitives individuelles.

Dans cette perspective, cette contribution s'articule en trois temps. Nous explicitons tout d'abord le concept de représentation sociale et indiquons en quoi il est pertinent dans le champ du « repreneuriat » $(\S 1)$. Puis nous montrons comment il permet de mieux appréhender les difficultés rencontrées par les différents acteurs, en amont du processus de reprise (\$2), puis en aval (§3). Pour assurer la réussite d'une reprise externe, il est en effet nécessaire que les différents acteurs prennent conscience de l'existence éventuelle d'un écart de représentation (sur l'entreprise à reprendre, les compétences à posséder, le mode de management requis...) et ce, le plus en amont possible du processus. Les acteurs doivent ensuite être en capacité de faire évoluer leurs représentations, afin de réduire cet écart qui peut s'avérer paralysant. Lorsque cela est possible, nous indiquons les facteurs qui nous paraissent faciliter cette évolution.

\footnotetext{
${ }^{2}$ Les quelques auteurs s'étant préoccupés des aspects psychologiques de la transmission sont principalement francophones. La plupart des travaux anglo-saxons portent en effet sur la transmission familiale. La transmission externe semble être une spécificité française.
} 


\section{I- LES REPRESENTATIONS SOCIALES AU SEIN DES APPROCHES SOCIOCOGNITIVES}

Nous définirons tout d'abord le concept de représentations sociales au sein des approches sociocognitives $(\$ 1.1)$, avant de montrer comment il permet de faire progresser les approches cognitives utilisées en entrepreneuriat et en repreneuriat (\$1.2).

\section{1/ Le concept de représentation sociale}

Le concept de représentation utilisé ici se fonde sur l'approche sociocognitive des organisations. Dans cette approche, et de façon simplifiée, on considère que les actions individuelles et collectives sont guidées par la signification que l'on attribue aux événements, ces actions façonnant à leur tour une réalité que l'on va interpréter et avec laquelle on va composer (Cossette, 2004). Les pratiques des acteurs sont vues comme intentionnelles et comme faisant l'objet d'activités réflexives. Dans cette littérature, on utilise souvent quasiindifféremment les notions de représentation, de schème et de cartes causales pour désigner la structure qui relie les idées entre elles par des liens de causalité. C'est cette structure cognitive de l'individu qui va jouer un rôle déterminant sur sa perception de la réalité extérieure et dans le diagnostic qu'il pose d'une situation problématique (Dearborn et Simon, 1958). Ces $a$ priori établis par le système de pensée permettent aux individus de gagner en rapidité dans leur prise de décision, en particulier lors de situations non-familières.

Les représentations sont ensuite dites « sociales » dans le sens où elles se constituent au cours d'interactions entre les individus (Moscovici, 1961 ; Jodelet, 1989 ; Doise, 1990). L'action n'est pas considérée uniquement comme le produit des activités cognitives, mais comme celui des interactions sociales. Ces interactions produisent des processus cognitifs, mais aussi psychologiques et sociologiques (croyances du sujet, jeu des régulations sociales). La figure 1 ci-dessous explicite graphiquement ces interactions entre les représentations, le contexte vécu et les actions des individus. 
$\underline{\text { Figure } \mathrm{n}^{\circ} 1 \text { : la relation représentation sociale-action }}$

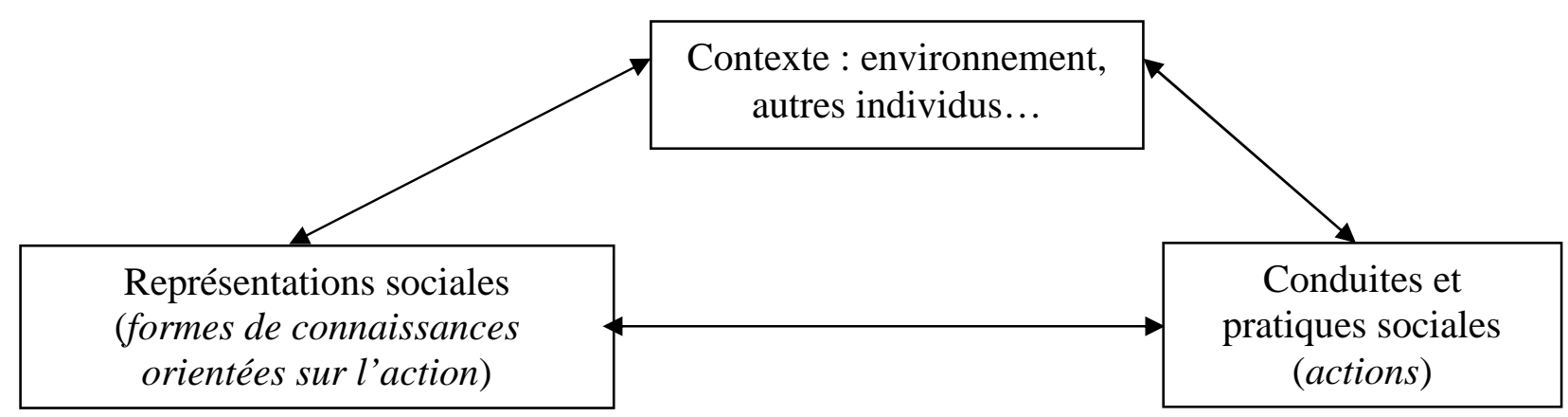

Légende : les flèches matérialisent les influences; elles sont à double sens à cause des phénomènes de rétroaction qui peuvent parfois s'opérer de façon quasi-simultanée.

(Adapté de Lauriol, 1998)

La représentation sociale est, en outre, toujours une représentation de quelque chose par quelqu'un, dans un rapport de symbolisation et d'interprétation de l'objet. Elle porte donc toujours sur un objet, au sens large, cet objet pouvant être humain, social, idéel ou matériel (une personne, une chose, un événement matériel, psychique ou social, une idée...).

Une fois forgée, cette représentation peut ensuite plus ou moins évoluer. La littérature fait en effet état d'une certaine dualité de la représentation, qui comporte à la fois des éléments stables (Abric ${ }^{3}$, 1989, 1994), appelés parfois noyaux ou éléments centraux, et des éléments instables (ou périphériques), en cours d'élaboration ou de modification. Ainsi l'adaptation d'une personne à son environnement serait non seulement régulée par ses représentations sociales, mais aussi limitée par la plasticité ${ }^{4}$ de ces dernières.

Enfin, rappelons que la représentation n'est pas uniquement individuelle, mais qu'elle peut également être collective, comme le montrent les travaux de Moscovici (1961) et de Jodelet (1989) sur les représentations sociales propres à une société donnée, ou les recherches sur les représentations organisationnelles (Weick et Bougon, 1986).

$\mathrm{Au}$ final, en reprenant pour partie la définition de Jodelet (1989), nous considérons qu'une représentation sociale est une forme inconsciente de connaissance, portée sur un objet par des acteurs sociaux (individuels et/ou collectifs). Cette connaissance est élaborée au cours de processus d'échanges et d'interactions. En tant que système d'interprétation, la représentation régit notre relation au monde et aux autres, oriente et organise nos conduites. De ce fait, nous

\footnotetext{
${ }^{3}$ Cet auteur utilise l'expression « schéma de représentation ».

${ }^{4}$ La notion de plasticité renvoie à la malléabilité de l'acteur, à sa capacité à évoluer.
} 
considérons que les représentations sociales constituent un outil méthodologique pertinent pour aborder la vie des organisations dans sa complexité, en fournissant une clé d'analyse et de compréhension des modes de pensée des individus, et donc des raisons de leurs décisions, actions, comportements et approches des situations rencontrées.

\section{2/ L'utilisation du concept de représentation sociale dans le champ de l'entrepreneuriat et du repreneuriat}

L'une des principales caractéristiques de l'entrepreneuriat (et du repreneuriat) étant qu'il s'agit d'un processus porté par un individu, il paraît indispensable de progresser dans la compréhension de ses composantes cognitives. Cependant, si des travaux récents montrent notamment tout le potentiel de l'approche cognitive pour répondre aux questions du «pourquoi » en entrepreneuriat (Baron, 2004) ou pour expliquer les erreurs commises par les entrepreneurs du fait des biais cognitifs et de l'utilisation d'heuristiques (Busenitz et Barney, $1997^{5}$ ), il existe à ce jour très peu de travaux mobilisant le concept de représentation sociale. Pourtant, pour Verstraete (2001), les représentations constituent les fondements de la vision stratégique qui permet à l'entrepreneur de s'engager avec son organisation vers le futur souhaité. Des travaux comme la thèse de Bornard (2007) sur les liens entre représentations et conduite du processus de création d'entreprise, ou l'ouvrage collectif consacré aux «représentations entrepreneuriales » et dirigé par Filion et Bourion (2008) semblent amorcer un intérêt accru pour cette approche. Ainsi Filion, dans son éditorial, indique que, de son point de vue, "ces approches sont fondamentales pour permettre aux acteurs entrepreneuriaux de mieux structurer leur pensée, en vue de définir plus adéquatement leur système d'activités et d'améliorer leurs pratiques » (p.13). De même, Cuzin et Fayolle (2005, p.13), dans un article consacré à l'exploration des différentes pratiques de l'accompagnement entrepreneurial, plaident pour la prise en compte de «tout ce qui relève des domaines de la visualisation et de la représentation de décisions et de situations complexes (...) pour apporter un outillage de nature à rendre plus efficient le travail sur les perceptions mentales ».

\footnotetext{
${ }^{5}$ Les heuristiques sont définies comme des stratégies simplifiées, utilisées en particulier dans des situations complexes, lorsque des informations moins complètes ou incertaines sont disponibles. Ainsi, la surcharge informationnelle, le haut degré d'incertitude et de nouveauté de la situation, le fort contenu émotionnel, la pression du temps et la fatigue sont autant de facteurs caractéristiques de la création d'une entreprise qui favorisent le risque de raisonnements inappropriés. Or on s'aperçoit que toutes les caractéristiques mentionnées ici se retrouvent également dans un processus de reprise d'entreprise.
} 
$\mathrm{Si}$, malgré son intérêt, on constate une faible utilisation du concept de représentation sociale dans le champ de l'entrepreneuriat, il n'existe à ce jour aucun travail le mobilisant dans le cadre du repreneuriat, alors même qu'il y paraît encore plus pertinent. En effet, la reprise est une aventure entrepreneuriale spécifique où l'on doit s'adapter à une organisation existante (contrairement à l'entreprise en création ; Bernard et Paliard, 2009). L'entreprise transmise est donc un objet sur lequel les acteurs peuvent avoir des représentations différentes. En outre, la reprise externe est un processus dans lequel interviennent de multiples interactions entre des acteurs variés et où s'entrecroisent des histoires individuelles, familiales et de groupes sociaux, chargées de mémoire et de vécu, engageant les acteurs au-delà de la composante économique. Elle fait enfin figure de processus où l'enjeu financier et social est souvent plus important que dans la création ex nihilo, étant données la taille moyenne supérieure de l'entreprise reprise et ses répercussions sur les différentes parties prenantes de l'opération (le maintien d'emplois et de l'activité économique de fournisseurs et/ou de clients étant souvent en jeu).

Selon nous, l'approche par les représentations permet de mieux cerner les blocages pouvant se produire à chaque étape du processus repreneurial. Ce processus peut en effet être découpé en trois ou quatre phases, selon l'existence ou non d'un passage de relais entre cédant et repreneur (phase qualifiée de «transition »; cf. figure $\mathrm{n}^{\circ} 2$ ). Dans un souci de simplification, nous parlerons de phases «amont» pour les étapes menant à la signature de la vente et de phases «aval » lorsque cette dernière a eu lieu. Les paragraphes qui suivent proposent une relecture des principales difficultés rencontrées en amont et en aval du processus repreneurial à travers le concept de représentation sociale. Pour chaque stade, nous précisons la nature de la représentation (individuelle ou collective), l'objet sur lequel elle porte et sa source (comment elle a été forgée). Puis nous expliquons comment ces représentations peuvent être l'origine de blocages et sous quelles conditions elles peuvent évoluer. 
CEDANT :

Préparation à la cession et choix du type de repreneur

\section{PREPARATION A LA} TRANSMISSION

REPRENEUR :

Préparation (psychologique et financière) à la reprise, recherche de l'entreprise à reprendre

\section{SIGNATURE}

TRANSITION?

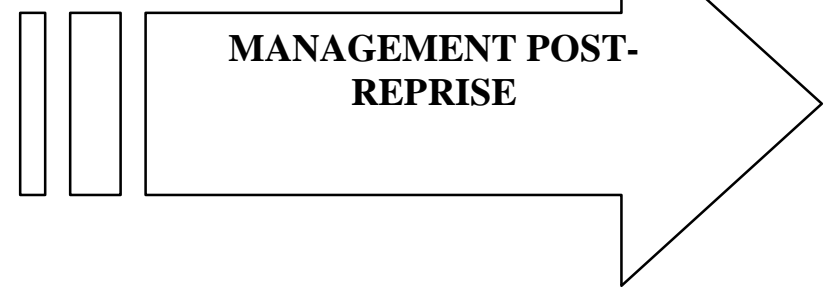

(Adapté de Picard et Thévenard-Puthod, 2004)

\section{II - LE ROLE DES REPRESENTATIONS SOCIALES EN AMONT DU PROCESSUS REPRENEURIAL}

En amont du processus, les représentations vont jouer un rôle à trois niveaux. Tout d'abord, le repreneur va devoir faire évoluer ses représentations sur l'entreprise idéalement recherchée et les compétences nécessaires au projet, pour mieux coller à la réalité du marché (\$2.1). En parallèle, le cédant va devoir lui aussi faire évoluer ses représentations sur le profil du repreneur souhaité, afin de se trouver un successeur (\$2.2). Enfin, les deux acteurs doivent parvenir à faire se «rapprocher » leurs représentations, afin que la transaction se réalise $(\S 2.3)$.

2.1/ Une évolution nécessaire de la représentation du repreneur : de l'entreprise rêvée à l'entreprise reprise 
Les repreneurs potentiels se lancent souvent dans le processus de reprise avec un certain nombre de représentations individuelles, qui concernent à la fois l'entreprise recherchée et les ressources et compétences nécessaires au déroulement de l'opération. Ces représentations se sont façonnées dans le contexte familial (présence ou non de chefs d'entreprise dans la famille, influence du statut social des parents sur une éventuelle volonté d'ascension sociale...) et au cours de l'expérience professionnelle de ces repreneurs (situations d'échec ou de réussite, rencontres avec des entrepreneurs...).

En premier lieu, les repreneurs vont entamer leur projet «repreneurial» avec des représentations de l'objet «entreprise à reprendre », dont les contenus peuvent varier d'un individu à l'autre. On relève en général trois grands types de situations (Deschamps et Paturel, 2009) induisant des contenus représentationnels différents :

- La reprise comme possibilité de se créer son emploi et de ne plus dépendre d'une hiérarchie parfois pesante : la représentation de l'entreprise à reprendre est une entreprise de petite taille (il ne s'agit pas de manager des salariés) et suffisamment pérenne pour assurer un salaire sur le long terme.

- La reprise comme levier d'accession à une position économique et sociale: la représentation de l'entreprise à reprendre est centrée sur la rentabilité potentielle devant permettre de dégager des revenus élevés.

- La reprise comme projet de réalisation personnelle : la représentation de l'entreprise à reprendre est celle d'une firme avec des potentialités de développement, idéalement une moyenne entreprise, structurée, dans laquelle le repreneur pourra se dégager de l'opérationnel.

En second lieu, les repreneurs vont avoir des représentations sur les ressources et compétences qu'il faut posséder pour réussir l'opération. Ainsi, les artisans considèrent souvent qu'il suffit de posséder des compétences techniques pour reprendre une activité artisanale (Picard et Thévenard-Puthod, 2004), croyance pendant longtemps véhiculée par la forte identité du Secteur des Métiers. A l'inverse, on voit également arriver de plus en plus de cadres repreneurs aux profils gestionnaires et managériaux, qui ont parfois fait l'objet de licenciement, et qui pensent pouvoir mettre à profit leur compétence managériale dans le cadre d'une reprise. Cette représentation concernant l'importance des compétences en management a pu être forgée au cours d'une carrière dans une grande entreprise.

Or ces différentes représentations concernant la cible idéale et les compétences requises ne correspondent pas toujours à la réalité du marché. Ainsi, les «belles PME » recherchées par certains repreneurs (la reprise comme projet de réalisation personnelle) sont rares et trouvent 
généralement preneurs auprès de l'environnement direct du cédant. En outre, l'achat de ces entreprises de taille moyenne nécessite des capitaux importants que les repreneurs individuels n'ont pas souvent à leur disposition (Lecointre, 2008). Elles se vendent donc la plupart du temps par le biais d'opérations de croissance externe. Ensuite, les cadres diplômés issus de grandes entreprises doivent prendre conscience que la «transférabilité » de leur compétence managériale est loin d'être évidente, car si elle peut s'avérer utile dans des entreprises de 10 à 20 salariés, celles-ci sont rares (en France, seule une entreprise sur quatre a plus de cinq salariés). Ils doivent donc accepter de passer du statut de manager spécialisé pouvant s'appuyer sur une hiérarchie à celui de dirigeant solitaire et polyvalent, devant souvent participer à des tâches très opérationnelles (gestions administrative et commerciale, production...). Dans le même esprit, la maîtrise d'une compétence technique ne suffit pas non plus à assurer la réussite d'une entreprise reprise, même artisanale. Enfin, les repreneurs qui arrivent de métiers scientifiques où les modèles mentaux sont parfois dominés par la compréhension du réel, à partir d'une perspective objectiviste, ne correspondent pas forcément aux modes de gestion des PME qui peuvent nécessiter des modèles où la subjectivité, la relativité et la personnalisation règnent (Filion et Barion, 2008).

Le problème est que les repreneurs ne perçoivent pas toujours cette incompatibilité entre leurs représentations (sur l'objet entreprise à reprendre et sur les compétences nécessaires au projet) et le réservoir d'entreprises disponibles. Ils ont du mal à percevoir la réalité du marché telle qu'elle est, l'absence de rationalité dans leur démarche ne leur faisant pas percevoir les signaux contraires. Ils rejettent alors souvent la faute sur les canaux de communication et soulignent le manque d'information sur les offres disponibles et l'opacité du marché (Deschamps et Paturel 2009), alors même que les bases de données se sont largement développées au cours de ces dix dernières années.

On voit donc ici se dessiner un premier obstacle qui pourrait être levé par un accompagnement adéquat des repreneurs par les acteurs institutionnels (CCI, CMA, syndicats professionnels...) : faire prendre conscience aux repreneurs, le plus en amont possible du processus, de cet écart entre leurs représentations et le marché de la reprise, pour faire évoluer ces dernières et permettre à ces individus de partir à la recherche d'une cible plus en accord avec leurs compétences et la réalité du marché. 


\section{2/ Une évolution nécessaire des représentations du cédant concernant le repreneur rêvé (fils spirituel) et l'entreprise cédée}

Les cédants, de leur côté, abordent également le processus de transmission avec un certain nombre de représentations sociales individuelles, issues de leur parcours de chef d'entreprise, de leur vécu personnel et des interactions qu'ils ont pu avoir avec les différentes parties prenantes de leur environnement. Ces représentations concernent à la fois l'entreprise cédée et le profil du repreneur idéal.

Concernant l'entreprise cédée, on constate que les cédants ont tendance à se représenter leur entreprise comme un prolongement de soi, ou comme leur enfant. En effet, les structures transmises sont souvent de petite taille, les cédants sont en grande majorité les créateurs de leur entreprise (pour 67\% d'entre eux, selon Lecointre, 2008) et cette dernière porte parfois leur nom, ce qui favorise une très grande personnalisation de la structure transmise (Lansberg, 1999). Or les caractéristiques de cette représentation ne sont pas sans poser problème.

En premier lieu, la volonté de céder n'est pas toujours très prononcée. Le cédant peut en effet avoir le sentiment de «vendre son bébé » pour de l'argent. La rupture du lien avec cette «possession chérie» (Pailot, 1999) survient en outre généralement à un âge où le questionnement sur la fin de la vie se fait plus aigu. Elle sonne donc l'heure des bilans, voire de la vieillesse, et signifie une transition de vie majeure : le passage du statut de propriétairedirigeant d'une PME à celui de retraité. Or il est particulièrement difficile pour quelqu'un qui a été une personne de pouvoir d'abandonner ce dernier (Bruneau, 2002). Plusieurs sentiments de perte se conjuguent donc: perte de son pouvoir (Vatteville, 1994), de son empreinte (Muller, 1996), de sa légitimité et plus globalement de sens (Boussaguet, 2007). Ces sentiments entrainent inévitablement des réactions émotionnelles fortes ${ }^{6}$ qui peuvent se traduire par un perpétuel report de la transmission: le cédant ne se représente pas son entreprise comme un objet externe valorisable et donc transmissible ou s'accroche à elle, tend à négliger la phase de préparation et rend la vente très délicate.

En second lieu, cette représentation de l'entreprise cédée comme un prolongement de soi ou comme un enfant, influence fortement le choix du successeur. Ainsi lorsque le cédant se résout à transmettre son entreprise, il désire plutôt le faire à quelqu'un de proche, en priorité à sa famille $(81 \%$ des propriétaires souhaitent que leur entreprise reste dans la famille, selon

\footnotetext{
${ }^{6}$ Or il est reconnu que les émotions (Oaksford and al., 1996) jouent un rôle fort dans les processus cognitifs et donc dans la constitution et l'évolution des représentations mentales des cédants.
} 
Marshall et al, 2006). Il cherche une certaine « consonance» cognitive. Ceci correspond au souhait légitime du cédant de voir son œuvre se perpétuer à travers ses enfants. Cependant la transmission familiale est devenue largement minoritaire en France. On recense au contraire une part croissante de cessions à un tiers (Oséo BDPME 2005). Le cédant est donc souvent contraint de vendre à l'extérieur. Il lui faut alors trouver un «fils spirituel» capable de pérenniser l'œuvre d'une vie (Kets de Vries, 1985). Cette recherche de filiation symbolique se traduit par la volonté d'une certaine proximité avec le repreneur. Le cédant cherche quelqu'un qui partage ses représentations de l'entreprise et du métier. Il peut ainsi être particulièrement exigeant sur les compétences requises, celles-ci pouvant être techniques ou managériales. La représentation qu'a un cédant des caractéristiques que doit posséder le repreneur (qualification, personnalité, comportement) a, au final, une grande influence sur sa capacité à faire suffisamment confiance à un repreneur potentiel pour lui transmettre son entreprise.

Or, face à la réalité du marché, il lui faut bien souvent faire évoluer ses représentations. Le clone recherché existe en effet rarement. Là encore, un meilleur accompagnement du cédant par des acteurs extérieurs au processus (accompagnateurs institutionnels ou anciens chefs d'entreprises ayant réalisé un transfert), pourrait favoriser cette prise de conscience de l'écart entre situation rêvée et situation réelle et aider le cédant à transmettre plus facilement son entreprise.

En définitive, cette analyse des comportements des deux principaux acteurs de la reprise externe, lors des phases amont du processus, peut bénéficier de la transposition aux figures du cédant et du repreneur de l'approche conceptuelle de Bruyat (1993, axiome 5) à propos du créateur d'entreprise ex-nihilo. Ces deux acteurs agissent, décident et forment des projets dont les matrices sont les représentations qu'ils ont de leurs aspirations, ressources et compétences, et possibilités de l'environnement. Chacun d'eux ne pourra concrétiser son projet que si une zone de cohérence peut apparaître au sein de leurs représentations. Pour cela, un cheminement individuel est d'abord nécessaire de part et d'autre, puis une nouvelle zone de congruence doit être trouvée entre les représentations des deux acteurs lors de leur rencontre. La figure $n^{\circ} 3$ schématise ces évolutions nécessaires. 
Figure 3. Les représentations du cédant et du repreneur externe concernant leur projet de $\underline{\text { transmission-reprise }}$

Etape 1 : la nécessité d'un cheminement individuel
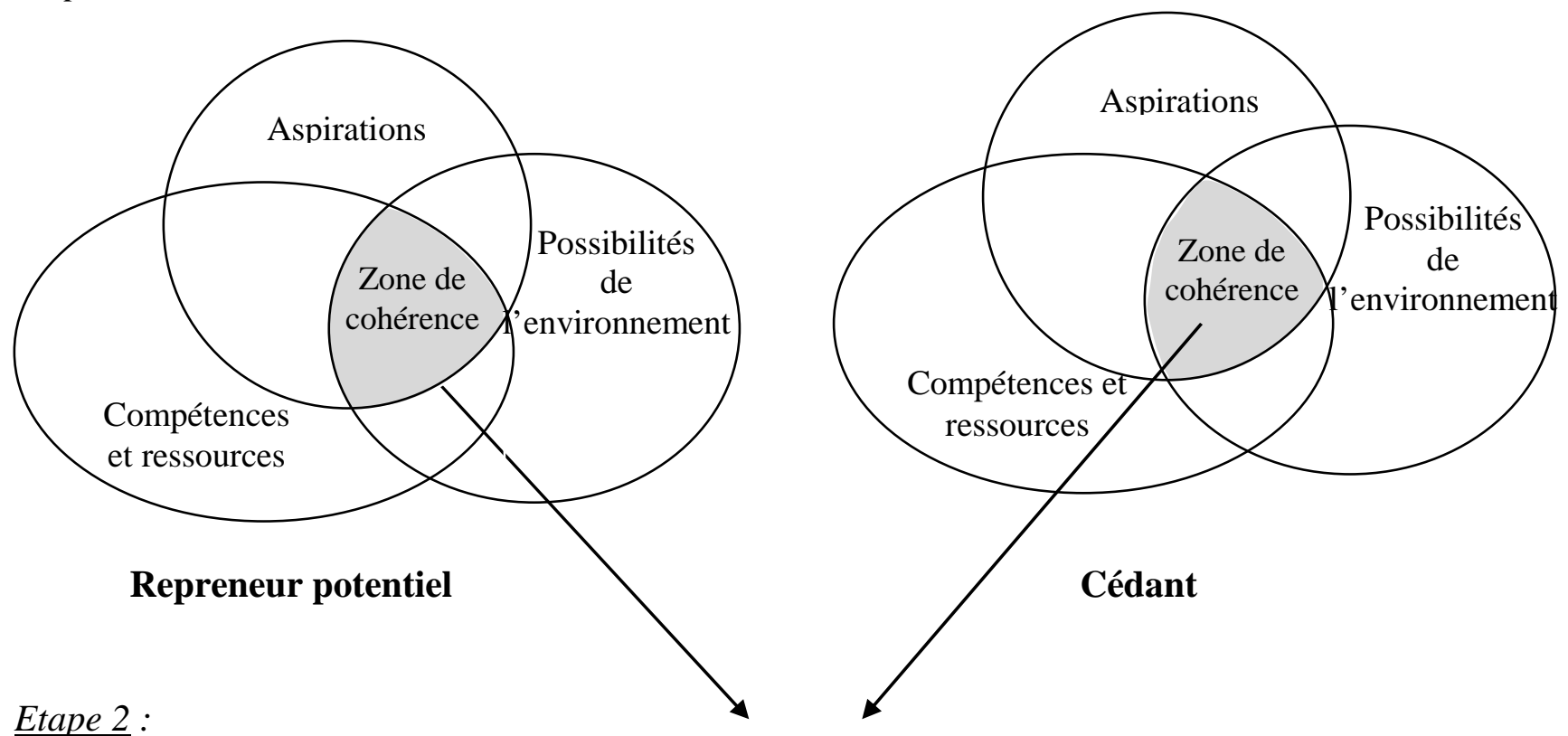

Rencontre : coïncidence des zones de cohérence des représentations

(Adapté de Bruyat, 1993, et de Deschamps et Paturel, 2009)

Ensuite, si ce rapprochement des représentations entre cédants et repreneurs est nécessaire pour que les premiers acceptent de transmettre leur «bébé » aux derniers, il se peut que de nouveaux achoppements se fassent ressentir au moment de la délicate phase de négociation du prix de vente.

\section{3/ L'influence des représentations du cédant et du repreneur au moment de la phase de négociation et d'accord final}

Cette phase du processus de transmission, bien que cruciale, n'a reçu que peu d'attention de la part des chercheurs en management, notamment parce qu'elle est particulièrement difficile à appréhender empiriquement (peu de cédants et/ou repreneurs permettent à des chercheurs de l'observer in vivo). Au cours de cette étape, les différences de représentations sociales vont là encore être individuelles, mais porter essentiellement sur l'objet à transmettre (l'entreprise). 
Les repreneurs doivent en effet parvenir à se faire une idée de ce que vaut réellement l'entreprise. Ils cherchent à faire un bon investissement et attendent de la part du cédant une transparence en matière de rentabilité, de pérennité et éventuellement de potentiel de développement de la structure. Les documents comptables renseignant sur la situation de l'entreprise doivent être disponibles et suffisamment clairs. Le cédant de son côté va pourtant avoir naturellement tendance à «maquiller la mariée » et à surévaluer son entreprise pour les raisons affectives déjà évoquées plus haut (il idéalise l'objet cédé). Il est en effet difficile d'évaluer objectivement une possession chérie qui représente l'accumulation de plusieurs années d'efforts, de succès et d'échecs, de capitaux investis... Le cédant peut en outre se sentir autant évalué que son entreprise (Bernard et Paliard, 2009). Chaque acteur agit donc non pas de manière rationnelle, même s'il croit le faire, en particulier lorsqu'il cherche à s'appuyer sur des analyses factuelles et normatives (analyse du marché, diagnostic financier, business plan,...), mais sous l'influence de ses schémas cognitifs.

En conséquence, les résultats de ces différences de représentations peuvent se traduire par des écarts importants dans les estimations du prix de l'entreprise, comme si on parlait de deux sociétés distinctes. La négociation du prix de vente peut alors donner lieu à une bataille acharnée, où les représentations des acteurs pèsent plus lourd que les critères objectifs d'évaluation. Elle peut également révéler des dissonances cognitives (Festinger, 1957), c'està-dire des mécanismes par lesquels le sujet cherche à réduire les incohérences qui le mettent en difficulté et qui peuvent faire évoluer ses représentations.

Dans cette étape, le recours à un arbitre disposant de davantage de recul et d'objectivité que les deux protagonistes (par exemple un spécialiste de l'évaluation) peut permettre à ces derniers de prendre conscience de ces écarts de représentations, préalable nécessaire à l'évolution de ces dernières. Ceci afin de parvenir plus facilement à un accord.

Si les acteurs parviennent finalement à s'entendre sur un prix de vente, alors la transaction sera effective. Cependant, la réussite d'une transmission ne se mesure pas à la signature de l'acte de vente. Le repreneur doit ensuite relever le défi du management post-reprise.

\section{III- LE ROLE DES REPRESENTATIONS SOCIALES EN AVAL DU PROCESSUS}

En aval du processus, les représentations vont également jouer un rôle clé. Elles vont tout d'abord influencer la qualité de la réalisation de la phase de transition entre le cédant et le repreneur (\$3.1). Elles vont ensuite affecter l'adhésion des salariés à ce nouveau dirigeant et à 
ses projets de développement, et la façon dont le repreneur va s'insérer dans l'environnement de l'entreprise (\$3.2).

\section{1/ L’influence des représentations sur le déroulement de la phase de transition}

Souvent présentée comme un gage de réussite d'une transmission ${ }^{7}$, cette phase de transition où cédant et repreneur cohabitent n'est pas sans poser de problèmes. Une alchimie qui ne s'établit finalement pas suffisamment entre les acteurs peut entraîner le départ anticipé du cédant. Si certains chercheurs utilisent la théorie du deuil pour matérialiser les difficultés ressenties par le cédant à se détacher de son entreprise (Bah, 2009), on peut néanmoins y voir encore ici un problème de différences de représentations, mais qui portent cette fois-ci sur le mode de management requis (par exemple la place du savoir-faire technique du dirigeant, le choix des pratiques GRH, l'utilisation de l'informatique...). Comme le soulignent Kets de Vries (1986) et Vatteville (1994), certains entrepreneurs acceptent difficilement une autre manière de gérer l'entreprise que la leur. Les représentations sociales du cédant sur le mode de management requis sont issues d'un travail de direction qui porte parfois sur de nombreuses années. Elles se sont constituées en interaction avec l'organisation (les salariés éventuels de l'entreprise) et l'environnement (les bonnes pratiques commerciales appréciées des clients par exemple). La représentation du repreneur sur ce que doit être un chef d'entreprise est, de son côté, également influencée par plusieurs facteurs : sa formation éventuelle au management, son environnement familial (présence ou non de chefs d'entreprises dans la famille), son expérience antérieure de dirigeant, mais aussi les représentations collectives en vigueur en France (ce que doit être un bon manager). Il peut ainsi exister un fossé générationnel et culturel entre un cédant autodidacte qui a l'âge de la retraite et un repreneur plus jeune ayant réalisé des études supérieures (à l'université ou dans une grande école) et/ou une carrière dans un grand groupe.

$\mathrm{Au}$ cours de ce passage de relais, le cédant doit parvenir à lâcher prise et à accepter cette différence de management. Mais le repreneur doit également faire preuve de capacité d'apprentissage et d'humilité pour réussir son adaptation à ce contexte qui lui est inconnu. Les deux acteurs doivent donc, là encore, être capables de faire évoluer leurs représentations. Il existe pour cela aujourd'hui relativement peu d'aide extérieure possible (certains cabinets de coaching tentent par exemple de se spécialiser sur l'accompagnement psychologique des

\footnotetext{
${ }^{7}$ Un dispositif national de tutorat a d'ailleurs été instauré dans ce sens (cf. lois du 2/08/05 et du 01/09/08 qui tendent à inciter un investissement plus systématique du cédant après la signature de la vente).
} 
cédants). Cette évolution ne peut par conséquent se faire que si les acteurs ont une personnalité suffisamment ouverte et empathique pour accepter ces différences de représentations et s'en accommoder.

\section{2/ L’influence des représentations dans le management post-reprise}

La phase de management post-reprise correspond au moment où le repreneur se retrouve seul à la tête de la structure acquise. Pour assurer la réussite de cette ultime phase du processus, il doit «pouvoir intégrer dans un ensemble cohérent, une panoplie de subjectivités parfois hétéroclites, tant à l'intérieur qu'à l'extérieur de l'entreprise, comprenant à la fois des collaborateurs, des fournisseurs et des clients aux valeurs et aux cultures souvent très différentes » (Filion et Bourion, 2008, p.16). L'échange de fonction entre le précédent dirigeant et le nouveau doit faire l'objet d'une légitimation auprès de l'ensemble des parties prenantes.

La reprise est non seulement un changement de personne, mais aussi une modification dans l'exercice du pouvoir. Un des enjeux forts de cette dernière phase du processus est donc d'obtenir l'adhésion des salariés. Sans cette dernière, de nombreux dysfonctionnements peuvent rapidement apparaitre (problèmes de motivation, de qualité du travail, voire démissions de salariés), nuisant à la santé de l'entreprise achetée (Savall et Zardet, 1995 ; Capet, 1998 ; Couteret, 1998). Le facteur humain est en effet le premier frein au changement (Henriet, 1999).

Or les salariés de l'entreprise reprise ont une représentation sociale collective de ce que doit être le chef d'entreprise. Dans certains cas, comme dans l'artisanat, le repreneur doit prouver sa légitimité à la tête de l'entreprise en montrant qu'il maîtrise parfaitement l'aspect technique du métier (Picard et Thévenard-Puthod, 2006). Dans d'autres, ce sont plutôt les compétences managériales et la capacité au leadership qui vont primer (Barach et al, 1988). Ces représentations vont notamment dépendre de l'histoire des salariés : elles se sont en partie construites au fil des interactions avec l'ancien patron, mais peuvent également provenir d'expériences antérieures. Or l'absence de ces dernières et une présence ancienne dans l'entreprise reprise rendent plus difficile l'évolution des représentations des salariés. Le personnel sera dans ce cas davantage attaché à son précédent leader et aux valeurs qu'ils privilégiaient (le dirigeant ayant une position centrale dans les PME; Mahé de Boislandelle, 1998). Le changement ne sera pas forcément bien attendu (Mouline 2000). L'évolution possible de ces représentations dépend toutefois également du contexte de la reprise, en 
particulier de la santé de l'entreprise. Ainsi, en s'inspirant des travaux de Pritchett (1985) portant sur la croissance externe, on peut distinguer différentes situations :

- Le sauvetage : transmission qui se fait dans l'urgence, au moment du décès du cédant par exemple, ou lors de difficultés financières importantes. Les salariés se représentent alors le repreneur comme un sauveur de l'entreprise. Toutefois cette prédisposition favorable peut évoluer vers une attitude moins constructive au fil des interactions, surtout si des sacrifices doivent être faits (fermetures de sites, licenciements de personnel).

- La collaboration, où la transmission se fait de façon plus progressive, avec un cédant qui a trouvé un repreneur proche de lui dans ses représentations et qui a pris le temps de l'introduire auprès des différentes parties prenantes de l'entreprise. La convergence de représentations entre le cédant et le repreneur, entamée en amont du processus, influence largement son déroulement ex post. Cette congruence facilite l'intégration du repreneur au sein de l'entreprise reprise et envoie un signal fort aux salariés pour les inciter à faire évoluer leurs représentations et à mieux accepter ce nouveau dirigeant. Toutefois, même si les premières interactions sont déterminantes (attitudes du repreneur, discours, premières réalisations), cette évolution nécessite du temps, les études portant sur les successions familiales montrant qu'il faut entre deux et quatre ans en moyenne pour qu'un successeur développe sa crédibilité auprès de l'organisation (Barach et al, 1988).

- La situation contestée, correspond enfin à un cas où le cédant a fini par vendre son entreprise par dépit, à un repreneur dont il ne partageait pas les représentations, et avec lequel il n'a pas réussi à s'entendre suffisamment pour prendre le temps de le légitimer aux yeux des différentes parties prenantes. Ici, les représentations des salariés portant sur ce que doit être un chef d'entreprise, trop liées à celles de l'ancien dirigeant, peuvent devenir un héritage empoisonné.

On peut par ailleurs répéter cette même analyse avec les parties prenantes externes de l'entreprise. Les relations entre un dirigeant de PME et ses partenaires extérieurs étant en général fortement personnalisées (Julien et Marchesnay, 1998; Torrès, 2007), les représentations des repreneurs sur le mode de management requis risquent d'être fort différentes de celles de ces derniers (clients, fournisseurs, banquiers...). Ceci peut être pénalisant dans le cadre des relations jugées stratégiques où une évolution est alors nécessaire. Or les représentations de ces acteurs sont influencées par les interactions antérieures qu'ils ont pu avoir avec le cédant. Dans certains cas, les interactions avec l'entreprise n'ont concerné que le personnel, ce qui facilite l'intégration du repreneur et l'application de son nouveau 
mode de management. Dans d'autres, où l'ancien dirigeant était l'interlocuteur central des acteurs de l'environnement, l'évolution des représentations sera plus délicate.

La figure $n^{\circ} 4$ schématise les différences de représentations qui s'exercent au cours des phases aval du processus de transmission externe. Elle montre notamment l'influence du «fantôme du cédant » qui continue de hanter l'entreprise reprise à travers l'emprise qu'il a eue sur les représentations des salariés et des parties prenantes externes.

$\underline{\text { Figure } n^{\circ} 4 \text { - Les différences de représentations }}$ $\underline{\text { sur ce que doit être le chef de l'entreprise transmise }}$

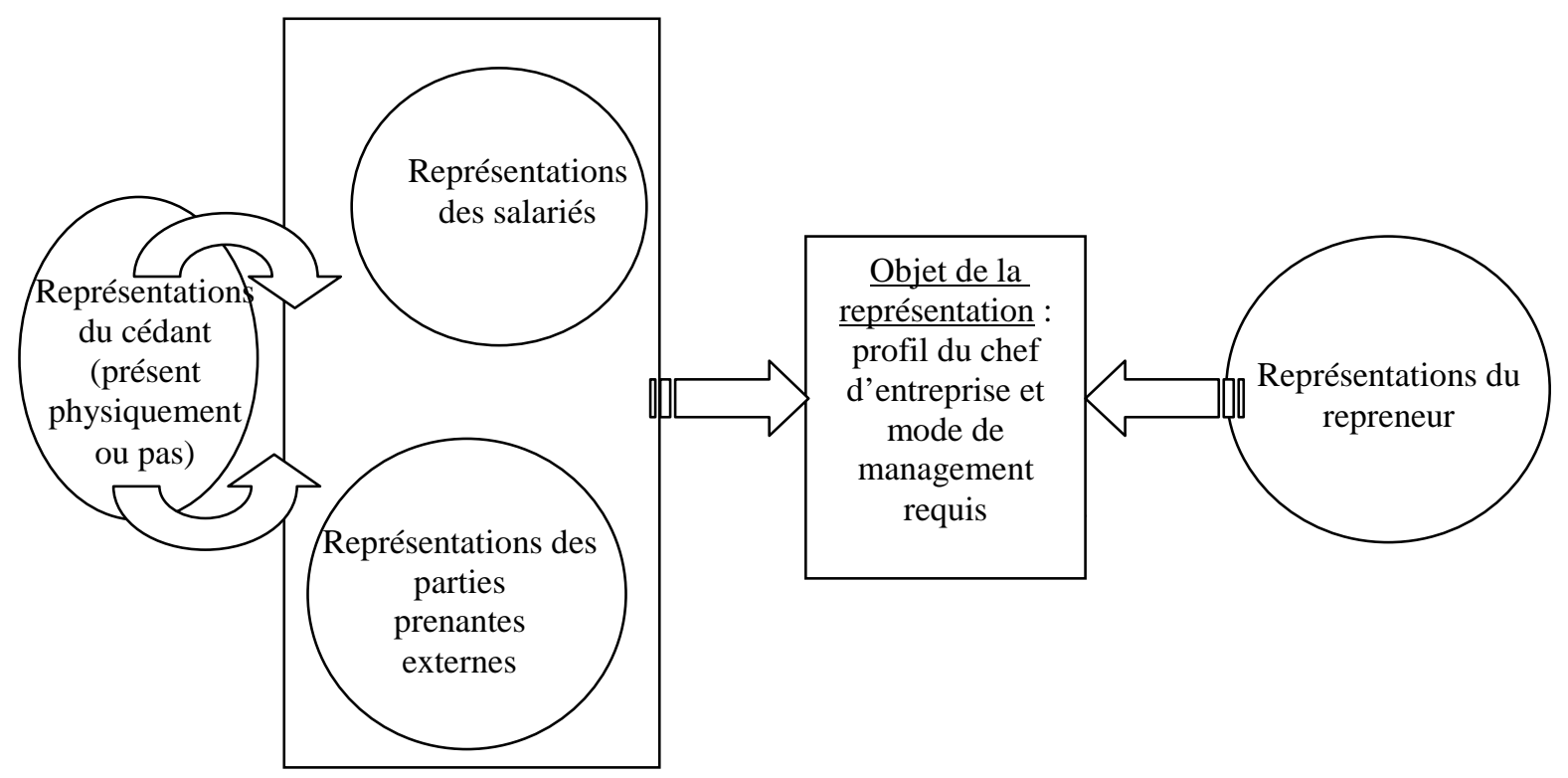

Il est ici plus difficile de proposer des solutions d'accompagnement aux différentes parties prenantes (internes et externes) pour qu'elles modifient leurs représentations. C'est le cédant qui, par sa propension à faire évoluer ses propres représentations et à accepter ce nouveau dirigeant, peut tenter d'impulser un changement chez les acteurs qu'il influençait. Mais c'est surtout le repreneur qui doit aller à la rencontre de ces différentes parties prenantes, susciter la parole et être suffisamment à l'écoute pour percevoir ces écarts de représentations. Une fois ceux-ci identifiés, il pourra plus facilement faire partager ses propres représentations. Le recours à une aide extérieure, sous la forme d'un tutorat post-reprise, pourrait également favoriser cette prise de recul et cette appréhension des représentations de ce corps social existant. 
En définitive, le tableau $\mathrm{n}^{\circ} 1$ de la page suivante synthétise le rôle joué par les représentations sociales (individuelles ou collectives), tout au long du processus de reprise. Il reprend ainsi, pour chaque étape, les acteurs concernés par les écarts de représentations et l'objet sur lequel ces derniers portent. Il indique également comment ces écarts peuvent paralyser le processus et quels sont les facteurs ou dispositifs d'accompagnement pouvant faciliter leur réduction. 
$\underline{\text { Tableau } \mathrm{n}^{\circ} 1 \text { : synthèse de l'influence exercée par les représentations dans le déroulement d'un processus de reprise externe }}$

\begin{tabular}{|c|c|c|c|c|c|}
\hline \multicolumn{2}{|c|}{ Phase du processus } & \multirow{2}{*}{$\begin{array}{c}\text { Acteurs } \\
\text { concernés }\end{array}$} & \multirow{2}{*}{$\begin{array}{l}\text { Objet sur lequel porte la différence } \\
\text { de représentations des acteurs } \\
\text { - Entreprise à reprendre «rêvée » } \\
\text { Compétences nécessaires au projet }\end{array}$} & \multirow{2}{*}{$\begin{array}{c}\text { Conséquences de ces différences de } \\
\text { représentations } \\
\text { - Incapacité à trouver une cible et non passage } \\
\text { à l'acte. Rejet de la faute sur « l'opacité du } \\
\text { marché » } \\
\text { - Incapacité à se résoudre à transmettre et à } \\
\text { faire confiance à un repreneur externe }\end{array}$} & \multirow{2}{*}{$\begin{array}{c}\text { Facteurs ou dispositifs d'accompagnement } \\
\text { facilitant la plasticité et l'évolution des } \\
\text { représentations } \\
\text { Accompagnement adéquat par un tiers (CCI, CMA, } \\
\text { club de repreneurs...) }\end{array}$} \\
\hline Amont & $\begin{array}{l}\text { Préparation de } \\
\text { la transmission }\end{array}$ & & & & \\
\hline & $\begin{array}{l}\text { Négociation et } \\
\text { accord }\end{array}$ & $\begin{array}{l}\text { Repreneur } \\
\text { Cédant }\end{array}$ & $\begin{array}{l}\text { L'entreprise cédée (sa valeur, son } \\
\text { potentiel) }\end{array}$ & $\begin{array}{l}\text { Impossibilité de se mettre d'accord sur un prix } \\
\text { et des clauses de vente }\end{array}$ & $\begin{array}{l}\text { Accompagnement adéquat par un tiers (spécialiste de } \\
\text { l'évaluation, expert-comptable...) }\end{array}$ \\
\hline \multirow[t]{2}{*}{ Aval } & Transition & $\begin{array}{l}\text { Repreneur } \\
\text { Cédant }\end{array}$ & $\begin{array}{c}\text { Le mode de management requis } \\
\text { (prédominance de la technique vs du } \\
\text { management / tradition vs } \\
\text { modernité...) }\end{array}$ & $\begin{array}{l}\text { Cohabitation et transmission de savoir-faire } \\
\text { impossible }\end{array}$ & $\begin{array}{l}\text { Personnalité des deux acteurs (capacité à lâcher prise } \\
\text { pour le cédant } v s \text { capacité d'humilité et } \\
\text { d'apprentissage pour le repreneur ; empathie). } \\
\text { Recours possible à un coach. }\end{array}$ \\
\hline & $\begin{array}{l}\text { Management } \\
\text { post-reprise }\end{array}$ & $\begin{array}{l}\text { Repreneur } \\
\text { Salariés } \\
\text { Parties } \\
\text { prenantes } \\
\text { externes }\end{array}$ & $\begin{array}{l}\text { Le profil du repreneur (ce qu'il doit } \\
\text { être, ses compétences) et le mode de } \\
\text { management requis }\end{array}$ & $\begin{array}{l}\text { - Manque de légitimité du repreneur aux yeux } \\
\text { des différentes parties prenantes } \\
\text { - Démotivation et/ou départ des salariés }\end{array}$ & $\begin{array}{l}\text { - Personnalité du repreneur (capacité à écouter et à } \\
\text { prendre du recul). Tutorat post-reprise } \\
\text { - Présence récente des salariés dans l'entreprise et } \\
\text { existence d'expériences professionnelles antérieures. } \\
\text { Contexte de la reprise (sauvetage, collaboration...)) } \\
\text { - Faible niveau d'interaction entre le cédant et les } \\
\text { parties prenantes externes. } \\
\text { - Capacité du cédant à avoir fait évoluer ses propres } \\
\text { représentations }\end{array}$ \\
\hline
\end{tabular}




\section{Conclusion}

L'objectif de cet article était d'expliquer une partie des échecs des processus repreneuriaux en mettant en avant l'influence des représentations sociales des acteurs. Le concept de représentation sociale présente en effet l'intérêt de fournir un cadre explicatif permettant de revisiter l'ensemble du processus de reprise, en englobant tous les acteurs concernés et toutes les phases du processus.

Ainsi, en amont, cet article a pu pointer le potentiel du concept de représentation sociale si l'on veut comprendre sous quelles conditions la rencontre entre repreneurs et cédants peut avoir lieu: lorsque leurs représentations (sur l'entreprise à céder/reprendre et sur les compétences/profils du repreneur) entrent en congruence. Puis, en aval, nous avons présenté les freins découlant des écarts de représentations concernant un autre «objet» représentationnel: ce que doit être un «manager» d'entreprise. Cette différence de représentations intervient non seulement entre le cédant et le repreneur, au moment de la phase de transition, mais aussi lors de la dernière phase (le management post-reprise) et cette fois-ci au plan collectif, dans les relations entre le nouveau chef d'entreprise et ses salariés, ainsi qu'avec l'ensemble des parties prenantes. Que le cédant soit présent ou non dans une phase de transition, son influence (positive ou négative) dans les représentations des différentes parties prenantes (internes et externes) reste forte, car les relations précédemment établies au cours du temps ont contribué à l'élaboration progressive d'une représentation collective du chef d'entreprise, que vient remettre en cause l'intervention du repreneur.

En traitant à la fois des phases amont du processus, dans ses dimensions individuelles, et des phases aval (post-transaction), dans ses dimensions collectives, l'approche par les représentations nous paraît donc particulièrement fertile sur le plan théorique, afin de progresser dans notre compréhension des difficultés à lever pour améliorer les chances de réussite des reprises d'entreprise. Elle met en effet en exergue la nécessaire appréhension par les différents acteurs de ces écarts de représentations. C'est seulement après cette prise de conscience que les acteurs peuvent ensuite essayer de faire évoluer leurs représentations. Si ces dernières restent figées, il y a peu de chance que la transaction se réalise ou qu'elle puisse être considérée comme un succès (par la pérennisation de l'entreprise transmise).

$\mathrm{Au}$ niveau des implications managériales de ce travail et dans une perspective d'accompagnement des individus, nous pensons que l'important n'est pas tant d'accéder aux différents contenus des représentations, en utilisant par exemple des outils comme les cartes 
cognitives qui sont difficilement opérationnalisables par les non-chercheurs ${ }^{8}$, que de leur faire prendre conscience de l'existence de ces représentations et de leur rôle, le plus en amont possible. L'aptitude des repreneurs ou cédants à savoir s'entourer apparaît cruciale pour faire évoluer les représentations. Le cédant, acteur central du processus, puisqu'il joue toujours un rôle même quand la transaction est terminée, mérite toute l'attention des dispositifs d'accompagnement. Les accompagnants constituent des acteurs importants dans le vécu psychologique de la cession et peuvent à ce titre prendre mieux en compte les représentations initiales, afin de les faire évoluer (Sammut, 2003). Nous rejoignons ainsi Filion et Bourion (2008) pour qui : «la compréhension de soi et de sa relation avec les autres est devenue une dimension cruciale de l'évolution des personnes et de la progression professionnelle ». La capacité des acteurs à faire évoluer leurs représentations (processus d'apprentissage, de réflexivité) pourrait par conséquent être incluse dans les facteurs clés de réussite d'une reprise, au même titre que les compétences managériales ou les ressources financières.

En termes de prolongement, cette recherche cherchait à poser des premiers jalons concernant l'ensemble des phases de la reprise externe. Elle a pour ambition de se poursuivre par des études de cas permettant d'approfondir le contenu du cadre d'analyse ici élaboré, en ciblant notamment plus précisément chaque étape et en faisant mieux apparaître les facteurs susceptibles d'influencer la plasticité des représentations et donc de faciliter l'évolution de ces dernières. Ceci permettra de proposer des pistes de dispositifs de soutien complémentaires à ceux déjà existants.

\section{Bibliographie}

ABRIC, J.C. (1989), «L'étude expérimentale des représentations sociales, » in D. Jodelet, (s/d), Les représentations sociales, Paris, Presses Universitaires de France.

ABric, J.C. (1994), Pratiques sociales et représentations, Paris, Presses Universitaires de France, Collection Psychologie sociale.

BAH, T. (2009), «La transition cédant-repreneur. Une approche par la théorie du deuil », Revue Française de Gestion, n¹94, p. 123-148.

BARACH, J.A., J. GANitsky, J.A CARson. et B.A Doochin (1988), «Entry of the next generation : strategic challenge for family business», Journal of Small Business Management, vol 26, p. 49-56.

\footnotetext{
8 De plus, les cartes cognitives sont pertinentes pour aider les acteurs à identifier leurs références et à réfléchir à leur comportement, mais leur approche statique de la représentation ne permet pas de comprendre leur genèse ou leur fonctionnement (Mounod et al.,1995).
} 
BARON, R. (1998), «Cognitive mechanisms in entrepreneurship : why and when entrepreneurs think differently than other people», Journal of Business venturing, vol 13 n ${ }^{\circ}$, p. 275-284.

BARON, R. (2004), «The cognitive perspective: a valuable tool for answering entrepreneuship's basic “why” questions», Journal of Business venturing, vol 19, n², p. 221239.

Baumert, H. (1992), Succession dans la PME familiale, Paris, Les Editions d'Organisation, collection PME.

Bernard M.J. et R. PALIARD (2009), «La mue nécessaire du repreneur d'entreprise », in Entrepreneuriat, ouvrage dirigé par M. Coster, Paris, Pearson Education.

Berthoz, A. (2003), La décision, Paris, Editions Odile Jacob.

BORNARD, F. (2007), La représentation de l'objet Entreprise par son créateur: quelles influences sur le processus entrepreneurial?, Thèse de doctorat en Sciences de Gestion, Université de Savoie.

Boussaguet, S. (2007), «Réussir son entrée dans l'entreprise : le processus de socialisation du nouveau dirigeant », Economie et Société, n¹6, janvier, p.145-165.

Bruneau, J.P. (2002), Psychanalyse et entreprises : création, développement et transmission des PME, Paris, Presses du Management.

BRUYAT, C. (1993), Création d'entreprise : contributions épistémologiques et modélisation, Thèse de doctorat en Sciences de Gestion, Université Pierre Mendès France, Grenoble.

BUSENITZ, L. et J. BARNEY (1997), «Differences between entrepreneurs and managers in large organisations : biases and heuristics in strategic decision making», Journal of Business Venturing, 12, p. 9-30.

CAPET, M. (1998) «La confiance des salariés dans le patron », Economie et Société, Tome 32, n8-9, août-septembre, p. 155-168.

Chaineau, A. (1989), La transmission d'entreprises : enjeux et perspectives, Paris, Presses Universitaires de France.

Codol, J.P. (1989), «Vingt ans de cognition sociale », Bulletin de Psychologie, 42:390, p. $472-491$

Cossette, P. (2004), L'organisation. Une perspective cognitiviste, Laval, Les Presses de l'Université.

Couteret, P. (1998), «Gérer les ressources humaines de la petite entreprise par la confiance », Revue Internationale de la PME, vol 11, n²-3, p.95-111.

Cuzin, R. et A. FAYOlle (2005), «Les dimensions structurantes de l'accompagnement en création d'entreprise », La Revue des Sciences de Gestion, n ${ }^{\circ} 10$, p. 77-88 
DEARBORN, D.C. and H.A. SiMON (1958), «Selective perception : a note on the departmental identifications of executives? », Sociometry, n²1, p.140-144.

Deschamps, B. et R. PATUREL (2009), Reprendre une entreprise : de l'intention à l'intégration du repreneur, Paris, Dunod, collection Entrepreneurs.

Doise, W. (1992), «L'ancrage dans les études sur les représentations sociales ? », Bulletin de Psychologie, vol. 45, $\mathrm{n}^{\circ} 405$.

FESTINGER ,L. (1957), A theory of cognitive dissonance, Stanford, Stanford University Press. FILION, L.J. et C. BOURION (2008), «Les représentations entrepreneuriales », Numéro spécial de la Revue Internationale de Psychosociologie, vol. 14, n³2.

HaVeman, H.A. et M.V. KHAIRE (2006), «Survival beyond succession? The contingent impact of founder succession on organizational failure », Journal of Business Venturing, vol 19, p. 437-463.

HENRIET, B. (1999), «La gestion des ressources humaines face aux transformations organisationnelles », Revue Française de Gestion, juin-août, p 82-93.

Jodelet, D. (1989), Les représentations sociales, (s/d), Paris, Presses Universitaires de France.

JoDELET, D. (1991), «L'idéologie dans l'étude des Représentations Sociales », dans V. Aesbischer, J.P. Deconchy, R. Lipiansky, Idéologies et représentations sociale, Fribourg, DelVal.

Julien, P.A. et M. Marchesnay (1998), La Petite Entreprise, Paris, Vuibert.

KeTS De VRIES, M.F.R. (1985), « The dark side of entrepreneurship », Harvard Business Review, $\mathrm{n}^{\circ} 63$, p. 160-167.

LANDSBerg, I.S. (1999), Succeeding Generations: Realizing the dream of families in business, Harvard Business School Press, 379 p.

LAURIOL, J. (1998), La décision stratégique en action, une perspective socio-cognitive, Paris, L'Harmattan.

LeCoIntre, G. (2008), La transmission d'entreprise en pratique, Paris, Gualino éditeur.

LeHMAn, P.J. (1993), «Le financement de la transmission des PME », Revue Française de Gestion, n 95 , septembre-octobre, p. 116-121.

Mahe de Boislandelle, H. (1998), Gestion des Ressources Humaines dans les PME, Paris, Economica.

Marshall, J.P. Sorenson R., K Brigham, E. Wieling, A. Reifman et R.S. Wampler, (2006), «The paradox of the family firm CEO: owner age relationship to succession-related processes and plans», Journal of Business Venturing, vol 21, p. 348-368. 
Malone, S.-C. (1989), «Selected Correlates of Business Continuity Planning in the Family Business », dans C. E. Aronoff, J. H. Astrachan, et J. L. Ward (Eds.), Family Business Sourcebook II, , p. 61-69.

MeIER, O (2002), «Problèmes de succession dans les PME familiales : freins et résistance culturelle », Gestion 2000, n4, juillet-août, p. 109-126.

Mouline, J.P. (2000), «Dynamique de la succession managériale dans la PME familiale non cotée », Finance Contrôle Stratégie, vol 3 n¹, p 197-222.

MoscovicI, S. (1961), «La psychanalyse, son image et son public », Paris : PUF.

Muller, J. (1996), «Le sujet et le pouvoir », dans Jean Muller (coord.), Le pouvoir dans les organisations : Masques et Mouvances, Groupe d'Etudes, de Recherche et d'Intervention, Editions L'Harmattan, p. 15-69.

OAKSFORD, M., Moreris, F.,Grainger, B., WililiaMs, J.M.G. (1996), « Mood, reasoning, and central executive processes», Journal of Experimental Psychology : Learning, Memory, and Cognition, $\mathrm{n}^{\circ} 22$, p. 476-492.

OSEO BDPME (2005), La transmission des petites et moyennes entreprises ; l'expérience d'Oséo Bdpme, étude n6203.02, juin 2005.

PAILOT, P. (1999), «Freins psychologiques et transmission d'entreprise : un cadre d'analyse fondé sur la méthode biographique », Revue Internationale PME, vol 3, p.9-32.

PicARd, C. et Thevenard-Puthod C. (2004), «La reprise de l'entreprise artisanale: spécificités du processus et conditions de sa réussite »", Revue Internationale PME, Vol 17 n², p. 94-121.

Picard, C. et C. Thevenard-Puthod (2006), «Confiance et défiance dans la reprise d'entreprises artisanales », Direction et Gestion, Revue des Sciences de Gestion, ${ }^{\circ} 216$, maijuin, p. 99-113.

PRITCHETT, P. (1985), After the merger : managing the shockwaves, Homewood, Dow Jones Irwin.

RICHARD, J.F. (1990), Les activités mentales, Paris, Armand Colin.

SAmmut, S. (2003), «L'accompagnement de la jeune entreprise », Revue Française de Gestion, n¹44, mai-juillet, p. 153-164.

SAVALl, H. et V. ZARDET (1995), «Management socio-économique de l'entreprise ou comment régénérer confiance et performances », dans Bidault F., PY Gomez et G. Marion, Confiance, Entreprise et société, Editions Eska, p 163-180. 
TORRES O. (2007), «Approche descriptive de la spécificité de gestion des PME : le mix de proximité », dans Management des PME, sous la direction de L.J. Filion, Paris, Pearson Education France.

VAtTEViLle, E. (1994), "Le risque successoral", Revue Française de Gestion, n98, marsmai, p.18-27.

Verstraete, T. (2001), «Entrepreneuriat: modélisation du phénomène », Revue de l'Entrepreneuriat, vol. $1, \mathrm{n}^{\circ} 1$.

WeICK, K.E and BougOn, M.G. (1986), Organizations as cognitive maps : charting ways to success and failure, in H.P. Sims, Jr et D.A. Giola (s/d), The thinking organization : dynamics of organizational social cognition, San Francisco, Jossey-Bass, p.102-135. 